Commun. Korean Math. Soc. 29 (2014), No. 1, pp. 1-8

http://dx.doi.org/10.4134/CKMS.2014.29.1.001

\title{
CONGRUENCES ON ABUNDANT SEMIGROUPS WITH QUASI-IDEAL $S$-ADEQUATE TRANSVERSALS
}

\author{
Lili Wang AND Aifa Wang
}

\begin{abstract}
In this paper, we give congruences on an abundant semigroup with a quasi-ideal $S$-adequate transversal $S^{\circ}$ by the congruence pair abstractly which consists of congruences on the structure component parts $R$ and $\Lambda$. We prove that the set of all congruences on this kind of semigroups is a complete lattice.
\end{abstract}

\section{Introduction and preliminaries}

The multiplicative inverse transversals of a regular semigroup were first introduced by T. S. Blyth and R. B. McFadden in 1982 [1]. As an analogue of an inverse transversal, an adequate transversal, was introduced by EI-Qallali in [3]. Afterwards, J. F. Chen and X. J. Guo studied the subclass of abundant semigroups with adequate transversals in $[2]$ and $[6,7,8]$, respectively. The congruence on regular semigroups with inverse transversals was studied by L. M. Wang and X. L. Tang (see [10], [11], [12]). Recently, X. J. Kong and P. Wang gave the structure theory of abundant semigroups having quasi-ideal $S$-adequate transversals in [8]. In this paper, we give congruences on abundant semigroups having quasi-ideal $S$-adequate transversals by the congruence triple and prove that the set of all congruences on this kind of semigroups is a complete lattice.

Let $S$ be a semigroup. By $a \mathcal{R}^{*} b$ we mean that $x a=y a$ if and only if $x b=$ $y b$ for all $x, y \in S^{1}$. The relation $\mathcal{L}^{*}$ is defined dually. $\mathcal{R}^{*}$ is a left congruence and $\mathcal{L}^{*}$ is a right congruence on $S$. It is easy to show that if $a$ and $b$ are regular elements of $S$ then $a \mathcal{R}^{*} b$ if and only if $a \mathcal{R} b$. We call a semigroup $S$ abundant if each $\mathcal{R}^{*}$-class and each $\mathcal{L}^{*}$-class contains an idempotent. For the sake of convenience, for $a \in S$, we denote the typical idempotent $\mathcal{L}^{*}$-related to $a$ by $a^{*}$ and we use $a^{\dagger}$ to denote the idempotent which is $\mathcal{R}^{*}$-related to $a$. An abundant semigroup in which idempotents commute is called adequate. It is clear that regular semigroups are abundant and that inverse semigroups are adequate.

Received June 7, 2012; Revised August 2, 2013.

2010 Mathematics Subject Classification. 20M10.

Key words and phrases. abundant semigroup, adequate transversal, quasi-ideal transversal, congruence, congruence pair. 
Lemma $1.1([5])$. Let $e \in E(S)$ and $a \in S$. Then $e \mathcal{R}^{*} a$ if and only if $e a=a$ and for all $x, y \in S^{1}, x a=y a$ implies $x e=y e$.

Lemma 1.2 ([4]). A semigroup $S$ is adequate if and only if each $\mathcal{R}^{*}$-class and each $\mathcal{L}^{*}$-class contains a unique idempotent and the subsemigroup generated by $E(S)$ is regular.

It is easy to show that if $S$ is adequate and $a, b \in S$, then $a \mathcal{R}^{*} b$ if and only if $a^{\dagger}=b^{\dagger}$ and $a \mathcal{L}^{*} b$ if and only if $a^{*}=b^{*}$.

Lemma 1.3 ([4]). If $S$ is an adequate semigroup, then for all $a, b \in S,(a b)^{*}=$ $\left(a^{*} b\right)^{*}$ and $(a b)^{\dagger}=\left(a b^{\dagger}\right)^{\dagger}$.

Notice that we can then immediately deduce that for all $a, b \in S,(a b)^{*}=$ $(a b)^{*} b^{*}$ and $(a b)^{\dagger}=a^{\dagger}(a b)^{\dagger}$.

Let $S$ be an abundant semigroup and $U$ is an abundant subsemigroup of $S$. We say that $U$ is a $*$-subsemigroup of $S$ if

$$
\mathcal{L}^{*}(U)=\mathcal{L}^{*}(S) \cap(U \times U), \mathcal{R}^{*}(U)=\mathcal{R}^{*}(S) \cap(U \times U) .
$$

It can be show that $U$ is a *-subsemigroup of $S$ if and only if for all $a \in U$ there exist $e, f \in E(U)$ such that $e \in L_{a}^{*}(S), f \in R_{a}^{*}(S)$ (see [3]).

Suppose that $S^{\circ}$ is an adequate $*$-subsemigroup of an abundant semigroup $S$. $S^{\circ}$ is called an adequate transversal of $S$ if for any element $x \in S$, there exists a unique element $x^{\circ} \in S^{\circ}$ and idempotents $e, f \in E$, such that $x=e x^{\circ} f$, where $e \mathcal{L}^{*} x^{\circ \dagger}$ and $f \mathcal{R}^{*} x^{\circ *}$ for $x^{\circ \dagger}, x^{\circ *} \in E^{\circ}$. It is straightforward to show [3], that such $e$ and $f$ are uniquely determined by $x$.

A subset $S^{\circ}$ of $S$ is called a quasi-ideal of $S$ if $S^{\circ} S S^{\circ} \subseteq S^{\circ}$. An adequate transversal $S^{\circ}$ of an abundant semigroup $S$ is called quasi-ideal adequate transversal if $S^{\circ}$ is a quasi-ideal of $S$. It is well known that the set $I=\left\{e \in S: e e^{\circ}=e\right\}$ is a left regular band and $\Lambda=\left\{f \in S: f^{\circ} f=f\right\}$ is a right regular band, and they play an important role in the study of regular semigroups with inverse transversals. In [2], Chen introduced two important idempotent subsets $I$ and $\Lambda$ and though they play a role similar to those in the regular case, in general, they are not subsemigroups of $S$. Following [9], if $S^{\circ}$ is an adequate transversal of an abundant semigroup $S$ and $I, \Lambda$ are both subsemigroups of $S$, then $S^{\circ}$ is called an $S$-adequate transversal of $S$.

Lemma 1.4 ([2]). Let $S$ be an abundant semigroup with a quasi-ideal adequate transversal $S^{\circ}$. Then for any $x, y \in S$

(1) $\overline{x y}=\bar{x} f_{x} e_{y} \bar{y}$;

(2) $e_{x y}=e_{x}\left(\bar{x} f_{x} e_{y}\right)^{\dagger}$

(3) $f_{x y}=\left(f_{x} e_{y} \bar{y}\right)^{*} f_{y}$.

Lemma 1.5 ([8]). Let $S$ be an abundant semigroup with an adequate transversal $S^{\circ}$. If $S^{\circ}$ is a right ideal of $S$, then $f_{x} \in E^{\circ}$ for every $x \in S$ and $E=I$. Consequently, $f_{x}=\bar{x}^{*}$ and thus $x=e_{x} \bar{x}$.

Dually, if $S^{\circ}$ is a left ideal of $S$, then $e_{a} \in E^{\circ}$ for every $a \in S$ and $E=\Lambda$. Consequently, $e_{a}=\bar{a}^{+}$and thus $a=\bar{a} f_{a}$. 
Lemma 1.6 ([8]). Let $R$ be a quasi-adequate semigroup with a right ideal adequate transversal $S^{\circ}$ and $\Lambda$ a right normal band with a left ideal semilattice transversal $E^{\circ}$. Suppose that the set of idempotents of $S$ coincides with $E^{\circ}$. Let $\Lambda \times R \longrightarrow S^{\circ}$ described by $(\lambda, x) \longrightarrow \lambda * x$ be a mapping, such that for any $x, y \in R$ and for any $\lambda, \mu \in \Lambda$ :

(1) $(\lambda * x) y=\lambda *(x y)$ and $\mu(\lambda * x)=(\mu \lambda) * x$;

(2) if $x \in E^{\circ}$ or $\lambda \in E^{\circ}$, then $\lambda * x=\lambda x$;

(3) if $x_{1} \mathcal{R}^{*} x_{2}$ in $R$, then for all $\mu_{1}, \mu_{2} \in \Lambda^{1}, y_{1}, y_{2} \in R^{1}, y_{1}\left(\mu_{1} * x_{1}\right)=$ $y_{2}\left(\mu_{2} * x_{1}\right)$ if and only if $y_{1}\left(\mu_{1} * x_{2}\right)=y_{2}\left(\mu_{2} * x_{2}\right)$.

Define a multiplication on the set

$$
\Gamma \equiv R|\times| \Lambda=\left\{(x, \lambda) \in R \times \Lambda: f_{x}=\lambda^{\circ}\right\}
$$

by

$$
(x, \lambda)(y, \mu)=\left(x(\lambda * y), f_{\lambda * y} \mu\right) .
$$

Then $\Gamma$ is an abundant semigroup with a quasi-ideal $S$-adequate transversal which is isomorphic to $S^{\circ}$.

Conversely, every abundant semigroup with a quasi-ideal S-adequate transversal can be constructed in this way.

Lemma $1.7([8])$. Let $(x, \lambda) \in \Gamma$. Denote $\mu=\left(e_{x}, \bar{x}^{\dagger}\right)$ and $\nu=\left(f_{x}, \lambda\right)$. Then $\mu, \nu \in E(\Gamma)$ and $\mu \mathcal{R}^{*}(x, \lambda) \mathcal{L}^{*} \nu$.

Lemma $1.8([8])$. Let $\left(x_{1}, \lambda_{1}\right),\left(x_{2}, \lambda_{2}\right) \in \Gamma$. Then

(1) $\left(x_{1}, \lambda_{1}\right) \mathcal{R}^{*}\left(x_{2}, \lambda_{2}\right)$ if and only if $e_{x_{1}}=e_{x_{2}}$ and ${\overline{x_{1}}}^{\dagger}={\overline{x_{2}}}^{\dagger}$.

(2) $\left(x_{1}, \lambda_{1}\right) \mathcal{L}^{*}\left(x_{2}, \lambda_{2}\right)$ if and only if $f_{x_{1}}=f_{x_{2}}$ and $\lambda_{1}=\lambda_{2}$.

\section{The main results}

In this section, we describe a congruence on abundant semigroups having a quasi-ideal $S$-adequate transversal. Let $S$ be a semigroup, $\mathcal{E}(S)$ the lattice of all equivalences on $S$. For any $\sigma \in \mathcal{E}(S)$, we say that $A \subseteq S$ a subset saturated by $\sigma$, if $A$ is a union of some $\sigma$-classes of $S$.

Suppose $\rho^{\Lambda}$ and $\rho^{R}$ are congruences on $\Lambda$ and $R$, respectively, and $\rho^{R}$ is saturated by $\mathcal{L}^{*}$ and $\mathcal{R}^{*}$. Then $\left(\rho^{R}, \rho^{\Lambda}\right)$ is called a congruence pair on $\Gamma$ if the following conditions hold:

(C.1) $\left.\rho^{R}\right|_{E^{\circ}}=\left.\rho^{\Lambda}\right|_{E^{\circ}}$;

(C.2) $(\forall \nu \in \Lambda)(\forall x, y \in R) x \rho^{R} y \Rightarrow(\nu * x) \rho^{R}(\nu * y)$;

(C.3) $(\forall z \in R)(\forall \lambda, \mu \in \Lambda) \lambda \rho^{\Lambda} \mu \Rightarrow f_{\lambda * z} \rho^{\Lambda} f_{\mu * z}$.

Define $\rho^{\left(\rho^{R}, \rho^{\Lambda}\right)}$ on $\Gamma$ by

$$
(x, \lambda) \rho^{\left(\rho^{R}, \rho^{\Lambda}\right)}(y, \mu) \Leftrightarrow x \rho^{R} y, \lambda \rho^{\Lambda} \mu .
$$

Theorem 2.1. Let $\Gamma$ be an abundant semigroup having a quasi-ideal S-adequate transversal as in Lemma 1.6, and $\left(\rho^{R}, \rho^{\Lambda}\right)$ be a congruence pair on $\Gamma$. Then $\rho^{\left(\rho^{R}, \rho^{\Lambda}\right)}$ is a congruence on $\Gamma$.

Conversely, every congruence on $\Gamma$ can be constructed in the above manner. 
Proof. Let $\left(\rho^{R}, \rho^{\Lambda}\right)$ be a congruence pair on $\Gamma$. Obviously, $\rho^{\left(\rho^{R}, \rho^{\Lambda}\right)}$ is an equivalence on $\Gamma$. Suppose that

$$
(x, \lambda),(y, \mu) \in \Gamma, \text { and }(x, \lambda) \rho^{\left(\rho^{R}, \rho^{\Lambda}\right)}(y, \mu) .
$$

Then

$$
x \rho^{R} y, \lambda \rho^{\Lambda} \mu .
$$

For any $(z, \nu) \in \Gamma$, by $x \rho^{R} y$ and C.2, we have

$$
(\nu * x) \rho^{R}(\nu * y) \text {. }
$$

Since $\rho^{R}$ is saturated by $\mathcal{L}^{*}$ and C.1, then

$$
f_{\nu * x}=(\nu * x)^{*} \rho^{\Lambda}(\nu * y)^{*}=f_{\nu * y} .
$$

It follows that

Hence

$$
z(\nu * x) \rho^{R} z(\nu * y) \text { and } f_{\lambda * z} \lambda \rho^{\Lambda} f_{\mu * z} \mu \text {. }
$$

That is,

$$
\left(z(\nu * x), f_{\lambda * z} \lambda\right) \rho^{\left(\rho^{R}, \rho^{\Lambda}\right)}\left(z(\nu * y), f_{\mu * z} \mu\right) .
$$

$$
(z, \nu)(x, \lambda) \rho^{\left(\rho^{R}, \rho^{\Lambda}\right)}(z, \nu)(y, \mu) .
$$

For any $(z, \nu) \in \Gamma$, by $\lambda \rho^{\Lambda} \mu$ and C.3, we have $f_{\lambda * z} \rho^{\Lambda} f_{\mu * z}$. Since $\lambda * z, \mu * z \in$ $S^{\circ}$, then

$$
f_{\lambda * z}=(\lambda * z)^{*}, f_{\mu * z}=(\mu * z)^{*} .
$$

By C.1, $(\lambda * z)^{*} \rho^{R}(\mu * z)^{*}$. Hence, by $\rho^{R}$ is saturated by $\mathcal{L}^{*},(\lambda * z) \rho^{R}(\mu * z)$. Furthermore, we have

$$
x(\lambda * z) \rho^{R} y(\mu * z) \text { and } f_{\lambda * z} \nu \rho^{\Lambda} f_{\mu * z} \nu .
$$

Hence

$$
\left(x(\lambda * z), f_{\lambda * z} \nu\right) \rho^{\left(\rho^{R}, \rho^{\Lambda}\right)}\left(y(\mu * z), f_{\mu * z} \nu\right) .
$$

Thus $(x, \lambda)(z, \nu) \rho^{\left(\rho^{R}, \rho^{\Lambda}\right)}(y, \mu)(z, \nu)$ and so $\rho^{\left(\rho^{R}, \rho^{\Lambda}\right)}$ is a congruence.

Conversely, assume that $\rho$ is a congruence on $\Gamma$. We define the following equivalences on $R$ and $\Lambda$, respectively,

$(\forall x, y \in R) x \rho_{R} y \Leftrightarrow\left(e_{x}, \bar{x}^{\dagger}\right) \rho\left(e_{y}, \bar{y}^{\dagger}\right) ;(\forall \lambda, \mu \in \Lambda) \lambda \rho_{\Lambda} \mu \Leftrightarrow\left(f_{x}, \lambda\right) \rho\left(f_{y}, \mu\right)$.

Since $\rho$ is a congruence on $\Gamma$, we have $\rho_{R}, \rho_{\Lambda}$ are equivalences on $R$ and $\Lambda$, respectively.

Let $(x, \lambda),(y, \mu),\left(x_{1}, \lambda_{1}\right),\left(y_{1}, \mu_{1}\right) \in \Gamma$. If $x \rho_{R} y$ and $x_{1} \rho_{R} y_{1}$, then

$$
\left(e_{x}, \bar{x}^{\dagger}\right) \rho\left(e_{y}, \bar{y}^{\dagger}\right) \text { and }\left(e_{x_{1}},{\overline{x_{1}}}^{\dagger}\right) \rho\left(e_{y_{1}},{\overline{y_{1}}}^{\dagger}\right) \text {. }
$$

Now we immediately get

$$
\left(e_{x}, \bar{x}^{\dagger}\right)\left(e_{x_{1}},{\overline{x_{1}}}^{\dagger}\right) \rho\left(e_{y}, \bar{y}^{\dagger}\right)\left(e_{y_{1}}, \bar{y}_{1}^{\dagger}\right) .
$$

And this implies that

Since

$$
\left(e_{x}\left(\bar{x}^{\dagger} * e_{x_{1}}\right), f_{\bar{x}^{\dagger} * e_{x_{1}}}{\overline{x_{1}}}^{\dagger}\right) \rho\left(e_{y}\left(\bar{y}^{\dagger} * e_{y_{1}}\right), f_{\bar{y}^{\dagger} * e_{y_{1}}}{\overline{y_{1}}}^{\dagger}\right) .
$$

$$
e_{x}\left(\bar{x}^{\dagger} * e_{x_{1}}\right) \overline{x x_{1}}=e_{x}\left(\bar{x}^{\dagger} * e_{x_{1}}\right) \bar{x}^{\dagger} \bar{x} f_{x} e_{x_{1}} \overline{x_{1}}=e_{x} \bar{x} f_{x} e_{x_{1}} \overline{x_{1}}=x x_{1}
$$


and

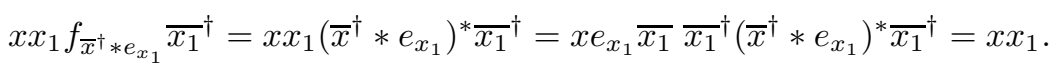

Then

$$
\left(e_{x x_{1}}, f_{\overline{x_{1}} * e_{x_{1}}} \bar{x}^{\dagger}\right) \rho\left(e_{y y_{1}}, f_{\bar{y}^{\dagger} * e_{y_{1}}}{\overline{y_{1}}}^{\dagger}\right) .
$$

So we have proved that $x x_{1} \rho_{R} y y_{1}$.

Suppose that $\lambda \rho_{\Lambda} \mu$ and $\lambda_{1} \rho_{\Lambda} \mu_{1}$, then we have

$$
\left(f_{x}, \lambda\right) \rho\left(f_{y}, \mu\right) \text { and }\left(f_{x_{1}}, \lambda_{1}\right) \rho\left(f_{y_{1}}, \mu_{1}\right) .
$$

Hence

$$
\left(f_{x}, \lambda\right)\left(f_{x_{1}}, \lambda_{1}\right) \rho\left(f_{y}, \mu\right)\left(f_{y_{1}}, \mu_{1}\right) .
$$

That is,

$$
\left(f_{x}\left(\lambda * f_{x_{1}}\right), f_{\lambda * f_{x_{1}}} \lambda_{1}\right) \rho\left(f_{y}\left(\mu * f_{y_{1}}\right), f_{\mu * f_{y_{1}}} \mu_{1}\right) .
$$

It follows from $f_{\lambda * f_{x_{1}}}=f_{\lambda * \lambda_{1}^{\circ}}=\lambda \lambda_{1}^{\circ}$ and $f_{\mu * f_{y_{1}}}=f_{\mu * \mu_{1}^{\circ}}=\mu \mu_{1}^{\circ}$ that

$$
\left(f_{x}\left(\lambda * f_{x_{1}}\right), \lambda \lambda_{1}^{\circ} \lambda_{1}\right) \rho\left(f_{y}\left(\mu * f_{y_{1}}\right), \mu \mu_{1}^{\circ} \mu_{1}\right) .
$$

Hence $\lambda \lambda_{1} \rho_{\Lambda} \mu \mu_{1}$.

And we have the following cases:

(1) $\left.\rho_{R}\right|_{E^{\circ}}=\left.\rho_{\Lambda}\right|_{E^{\circ}}$ is obvious. So (C.1) holds.

(2) Let $x, y \in R$ and $x \rho_{R} y$. Then

$$
\left(e_{x}, \bar{x}^{\dagger}\right) \rho\left(e_{y}, \bar{y}^{\dagger}\right) .
$$

Hence

$$
\left(f_{z}, \nu\right)\left(e_{x}, \bar{x}^{\dagger}\right) \rho\left(f_{z}, \nu\right)\left(e_{y}, \bar{y}^{\dagger}\right)
$$

That is,

$$
\left(f_{z}\left(\nu * e_{x}\right), f_{\nu * e_{x}} \bar{x}^{\dagger}\right) \rho\left(f_{z}\left(\nu * e_{y}\right), f_{\nu * e_{y}} \bar{y}^{\dagger}\right) .
$$

It follows from $f_{z}\left(\nu * e_{x}\right) \overline{(\nu * x)}=\nu^{\circ} \nu e_{x}(\nu * x)^{\dagger}(\nu * x)=(\nu * x)$ that

$$
\left(e_{\nu * x}, f_{\nu * e_{x}} \bar{x}^{\dagger}\right) \rho\left(e_{\nu * y}, f_{\nu * e_{y}} \bar{y}^{\dagger}\right) .
$$

Thus $(\nu * x) \rho_{R}(\nu * y)$ and so C.2 hold.

(3) Let $\lambda, \mu \in \Lambda$ and $\lambda \rho_{\Lambda} \mu$. Then

$$
\left(f_{x}, \lambda\right) \rho\left(f_{y}, \mu\right) .
$$

Hence

It follows that

$$
\left(f_{x}, \lambda\right)(z, \nu) \rho\left(f_{y}, \mu\right)(z, \nu) .
$$

$$
\left(f_{x}(\lambda * z), f_{\lambda * z} \nu\right) \rho\left(f_{y}(\mu * z), f_{\mu * z} \nu\right) .
$$

Thus $f_{\lambda * z} \nu \rho_{\Lambda} f_{\mu * z} \nu$ and so $f_{\lambda * z} \nu f_{z} \rho_{\Lambda} f_{\mu * z} \nu f_{z}$. Moreover, $(\lambda * z)^{*} z^{*} \rho_{\Lambda}(\mu * z)^{*} z^{*}$. Then $f_{\lambda * z} \rho_{\Lambda} f_{\mu * z}$ and so C.3 hold.

Let $x \rho_{R} y$. Then

$$
\left(e_{x}, \bar{x}^{\dagger}\right) \rho\left(e_{y}, \bar{y}^{\dagger}\right)
$$


From $x \mathcal{R}^{*} x^{*}, y \mathcal{R}^{*} y^{*}$, we have $e_{x}=e_{x^{*}}, e_{y}=e_{y^{*}}$. Since $e_{x}=e_{x} \cdot \bar{x}^{\dagger} \cdot \bar{x}^{\dagger}=e_{x^{*}}$, we have $\bar{x}^{\dagger}={\overline{x^{*}}}^{\dagger}$. Then

$$
\left(e_{x^{*}},{\overline{x^{*}}}^{\dagger}\right) \rho\left(e_{y^{*}},{\overline{y^{*}}}^{\dagger}\right) .
$$

It follows that $x^{*} \rho_{R} y^{*}$ and so $\rho_{R}$ is saturated by $\mathcal{R}^{*}$. Similarly, we can proved $\rho_{R}$ is saturated by $\mathcal{L}^{*}$.

Now from the above proof, $\left(\rho_{R}, \rho_{\Lambda}\right)$ is a congruence pair on $\Gamma$. have

By the direct part, $\rho^{\left(\rho_{R}, \rho_{\Lambda}\right)}$ is a congruence. If $(x, \lambda) \rho^{\left(\rho_{R}, \rho_{\Lambda}\right)}(y, \mu)$, then we

And hence

$$
x \rho_{R} y, \lambda \rho_{\Lambda} \mu \text {. }
$$

$$
\left(e_{x}, \bar{x}^{\dagger}\right) \rho\left(e_{y}, \bar{y}^{\dagger}\right),\left(f_{x}, \lambda\right) \rho\left(f_{y}, \mu\right) .
$$

From $\bar{x} \mathcal{R}^{*} \bar{x}^{*} \mathcal{R} f_{x} \mathcal{L}^{*} x$ and $\rho_{R}$ is saturated by $\mathcal{L}^{*}$ and $\mathcal{R}^{*}$, we have $\bar{x} \rho_{R} x \rho_{R} \bar{x}^{*}$. Similarly, we have $\bar{y} \rho_{R} y \rho_{R} \bar{y}^{*}$. Thus $\bar{x} \rho_{R} \bar{y} \rho_{R} \bar{x}^{*} \rho_{R} \bar{y}^{*}$. By $\bar{x}^{*} \rho_{R} \bar{y}^{*}$ and C.1, we have $\bar{x}^{*} \rho_{\Lambda} \bar{y}^{*}$. And hence

$$
\left(\bar{x}, \bar{x}^{*}\right) \rho\left(\bar{y}, \bar{y}^{*}\right)
$$

It follows from

$$
\left(e_{x}, \bar{x}^{\dagger}\right) \rho\left(e_{y}, \bar{y}^{\dagger}\right),\left(f_{x}, \lambda\right) \rho\left(f_{y}, \mu\right) \text { and }\left(\bar{x}, \bar{x}^{*}\right) \rho\left(\bar{y}, \bar{y}^{*}\right)
$$

that

$$
\left(e_{x}, \bar{x}^{\dagger}\right)\left(\bar{x}, \bar{x}^{*}\right)\left(f_{x}, \lambda\right) \rho\left(e_{y}, \bar{y}^{\dagger}\right)\left(\bar{y}, \bar{y}^{*}\right)\left(f_{y}, \mu\right)
$$

That is,

$$
(x, \lambda) \rho(y, \mu) \text {. }
$$

Thus, $\rho^{\left(\rho_{R}, \rho_{\Lambda}\right)} \subseteq \rho$. Since $\rho \subseteq \rho^{\left(\rho_{R}, \rho_{\Lambda}\right)}$ is obvious, $\rho^{\left(\rho_{R}, \rho_{\Lambda}\right)}=\rho$.

We denote the set of all congruences on $\Gamma$ and the set of all congruence pairs on $\Gamma$ constructed as in Theorem 2.1 by $C(\Gamma)$ and $C T(\Gamma)$.

Lemma 2.2. If $\left(\rho_{1}^{R}, \rho_{1}^{\Lambda}\right),\left(\rho_{2}^{R}, \rho_{2}^{\Lambda}\right) \in C T(\Gamma)$, then

$$
\rho^{\left(\rho_{1}^{R}, \rho_{1}^{\Lambda}\right)} \subseteq \rho^{\left(\rho_{2}^{R}, \rho_{2}^{\Lambda}\right)} \Leftrightarrow \rho_{1}^{R} \subseteq \rho_{2}^{R}, \rho_{1}^{\Lambda} \subseteq \rho_{2}^{\Lambda} .
$$

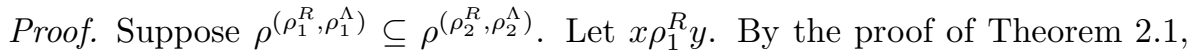
there exist $\left(e_{x}, \bar{x}^{\dagger}\right),\left(e_{y}, \bar{y}^{\dagger}\right) \in E(\Gamma)$ and $\rho_{1}, \rho_{2} \in C(\Gamma)$ such that

$$
\left(\left(e_{x}, \bar{x}^{\dagger}\right),\left(e_{y}, \bar{y}^{\dagger}\right)\right) \in \rho_{1} \subseteq \rho_{2} .
$$

Hence $x \rho_{2}^{R} y$, and immediately we get $\rho_{1}^{R} \subseteq \rho_{2}^{R}$. Similarly, we have $\rho_{1}^{\Lambda} \subseteq \rho_{2}^{\Lambda}$. The reverse implication is obvious.

Define $\leq$ on $C T(\Gamma)$ by

$$
\left(\rho_{1}^{R}, \rho_{1}^{\Lambda}\right) \subseteq\left(\rho_{2}^{R}, \rho_{2}^{\Lambda}\right) \Leftrightarrow \rho_{1}^{R} \subseteq \rho_{2}^{R}, \rho_{1}^{\Lambda} \subseteq \rho_{2}^{\Lambda} .
$$

Then $C T(\Gamma)$ is a partial ordered set with respect to $\leq$. By Theorem 2.1 and Lemma 2.2 , we can easily see that $C(\Gamma)$ and $C T(\Gamma)$ are isomorphic as partial ordered set. 
Proposition 2.3. Let $\Omega \subseteq C(\Gamma)$ and $T_{\rho}=\left(\rho^{R}, \rho^{\Lambda}\right)$ where $\rho \in \Omega$. Then

$$
T_{\left(\cap_{\rho \in \Omega} \rho\right)}=\left(\bigcap_{\rho \in \Omega} \rho^{R}, \bigcap_{\rho \in \Omega} \rho^{\Lambda}\right)
$$

and

$$
T_{\left(\bigvee_{\rho \in \Omega} \rho\right)}=\left(\bigvee_{\rho \in \Omega} \rho^{R}, \bigvee_{\rho \in \Omega} \rho^{\Lambda}\right) .
$$

Proof. The first equality is obvious, we only need to prove the second equality. Let $x, y \in R$ be such that $x\left(\bigvee_{\rho \in \Omega} \rho\right)^{R} y$. Then

$$
i=\left(e_{x}, \bar{x}^{\dagger}\right) \bigvee_{\rho \in \Omega} \rho\left(e_{y}, \bar{y}^{\dagger}\right)=j .
$$

Hence, there exist $\rho_{i} \in \Omega$ and $a_{i}=\left(e_{x_{i}}, \bar{x}_{i}^{\dagger}\right) \in \Gamma$ such that

$$
i \rho_{1} a_{1} \rho_{2} a_{2} \cdots a_{n-1} \rho_{n} j \text {. }
$$

This implies that

We have proved that

$$
x \rho_{1}^{R} x_{1} \rho_{2}^{R} x_{2} \cdots x_{n-1} \rho_{n}^{R} y
$$

$$
\left(\bigvee_{\rho \in \Omega} \rho\right)^{R} \subseteq\left(\bigvee_{\rho \in \Omega} \rho^{R}\right)
$$

$\left(\bigvee_{\rho \in \Omega} \rho^{R}\right) \subseteq\left(\bigvee_{\rho \in \Omega} \rho\right)^{R}$ is obvious. The dually equality can be proved similarly.

Now, by summing up the above results, we obtain the following theorem.

Theorem 2.4. Let $\Gamma$ be can constructed in Lemma 1.6. The $C T(\Gamma)$ forms a complete lattice with respect to $\leq$ and $C(\Gamma)$ is isomorphic to $C T(\Gamma)$ as complete lattice.

Acknowledgements The authors would like to thank the referee for his or her useful and valuable comments and suggestions relating to this paper.

\section{References}

[1] T. S. Blyth and R. B. McFadden, Regular semigroups with a multiplicative inverse transversal, Proc. Roy. Soc. Edinburgh Sect. A 92 (1982), no. 3-4, 253-270.

[2] J. F. Chen, Abundant semigroups with adequate transversals, Semigroup Forum 60 (2000), no. 1, 67-79.

[3] A. El-Qallali, Abundant semigroups with a multiplicative type A transversal, Semigroup Forum 47 (1993), no. 3, 327-340.

[4] J. B. Fountain, Adequate semigroups, Proc. Edinburgh Math. Soc. 22 (1979), no. 2, $113-125$.

[5] _ Abundant semigroups, Proc London Math. Soc. 44 (1982), no. 1, 103-129.

[6] X. J. Guo, Abundant semigroups with a multiplicative adequate transversal, Acta Math. Sin 18 (2002), no. 2, 229-244.

[7] X. J. Guo and L. M. Wang, Idempotent-connected abundant semigroups which are disjoint unions of quasi-ideal adequate transversals, Comm. Algebra 30 (2002), no. 4, 1779-1800. 
[8] X. J. Kong and P. Wang, Abundant semigroups with quasi-ideal S-adequate transversals, Commun. Korean Math. Soc. 26 (2011), no. 1, 1-12.

[9] X. L. Tang, Regular semigroups with inverse transversals, Semigroup Forum 55 (1997), no. $1,24-32$.

[10] X. L. Tang and L. M. Wang, Congruences on regular semigroups with inverse transversals, Comm. Algebra 23 (1995), no. 11, 4157-4171.

[11] L. M. Wang, On congruence lattice of regular semigroups with Q-inverse transversals, Semigroup Forum 50 (1995), no. 2, 141-160.

[12] L. M. Wang and X. L. Tang, Congruence lattices of regular semigroups with inverse transversals, Comm. Algebra 26 (1998), no. 4, 1234-1255.

LILI WANG

School of Mathematics and Statistics ChongQing University of Technology

Chongqing 400054, P. R. China

E-mail address: wllaf@163.com

AIfA WANG

School of Mathematics and Statistics

Chongqing University of Technology

Chongling 400054, P. R. China

E-mail address: wangaf1980@163.com 